\title{
Use of Reconfigurable IM Regions to Suppress Propagation and Polarization Dependent Losses in a MMI Switch
}

\author{
G. Singh, V. Janyani, P. Yadav
}

\author{
G. Singh, V. Janyani, V. Janyani \\ Department of Electronics and Communication Engineering \\ Malaviya National Institute of Technology Jaipur-India \\ E-mail: gschoudhary75@gmail.com, Tel.: +91-1412713431
}

\begin{abstract}
:
With this work, use of reconfigurable index modulated (IM) regions to accelerate the performance of a multimode interference (MMI) based photonic switch is presented. Appropriate dimension for such regions are defined to suppress the transition losses and to optimize the area coverage. It has been noticed that by reconfiguring the IM regions, perfect switching for test wavelengths of $1.3 \mu \mathrm{m}$ and $1.55 \mu \mathrm{m}$ with low insertion loss (I.L.) levels, $\leq 1.2 \mathrm{~dB}$ and excess loss (E.L.) levels, $\leq 0.17 \mathrm{~dB}$ can be achieved with vacillation of extremely low polarization dependent losses (PDLs), which are $\leq$ $0.15 \mathrm{~dB}$. For either case of input test wavelengths, generated crosstalk (CT) levels are found better than $-21.8 \mathrm{~dB}$ for TE and $-20.2 \mathrm{~dB}$ for TM polarization state.

Keywords: Selfimages in MMI waveguides, Reconfigurable IM regions, I.L. and E.L., CT levels.
\end{abstract}

\section{Introduction}

Image replication in the MMI waveguides strongly depends upon the refractive index changing properties of the waveguide material. Various materials such as polymers, $\mathrm{SiO}_{2}-\mathrm{SiON}, \mathrm{SOI}$, $\mathrm{LiNbO}_{3}$, III-V group semiconductors and their composites have been used as a backplane to develop multimode waveguides to implement optical couplers and switches. [1-9] Such waveguides are suitable to realize many optical devices utilizing their self-imaging principle and possess various characteristics such as compactness, relaxed fabrication tolerance, large optical bandwidth, and polarization insensitivity. [8-10] Design of ultra-short couplers and switches based on MMI waveguides have been achieved by eliminating photonic confinement to change their behaviors or by alteration in the imaging length with suitable index variance. $[5,6]$ In recent, researchers has shown a great interest for modeling of polarization independent photonic switches for wide wavelength spectrum, low losses and CT levels using MMI waveguiding structures. This article deals with modeling of a $2 \times 2 \mathrm{MMI}$-switch, which is tuned by integrated reconfigurable IM regions with its coupling area. The channel waveguides are optimized to reduce overall switch area coverage. The device has been evaluated for its satisfactory performance for operating test wavelengths of 1.3 Âlm and 1.55 Âlm. The contents of the article are as follows. A MMI-switch structure and its operation with an IM region are described in Section II. The detailed modeling and switching characteristics of the proposed structure are analyzed by finite difference beam propagation method (FD-BPM) in Section III, and then conclusions are summarized in Section IV.

\section{MMI-switch based on self-imaging principle}

The concept of self-imaging explains the replication of images of random objects in multimode waveguides. [10,11] The switching in MMI waveguides based switches can be achieved by modifying the refractive index at specific areas within the MMI waveguide, which are collocated 
with the occurrence of multiple self-images. [8] This change in the refractive index is the prime factor behind altering the phase relation between the self-images, which ultimately modifies the output image and switches the light between the output waveguides. MMI structures usually realize optical functions by tuning the refractive index of the image modulation region, located within the MMI section. Changes in the refractive indices of the segments (IM regions) inside the MMI section also bring variation in the effective width of the MMI region, thereby changes occurs in the beat length $\left(L_{\pi}\right)$.

In configuration shown by Fig. 1, the confinement guide regions are shown, which allows the

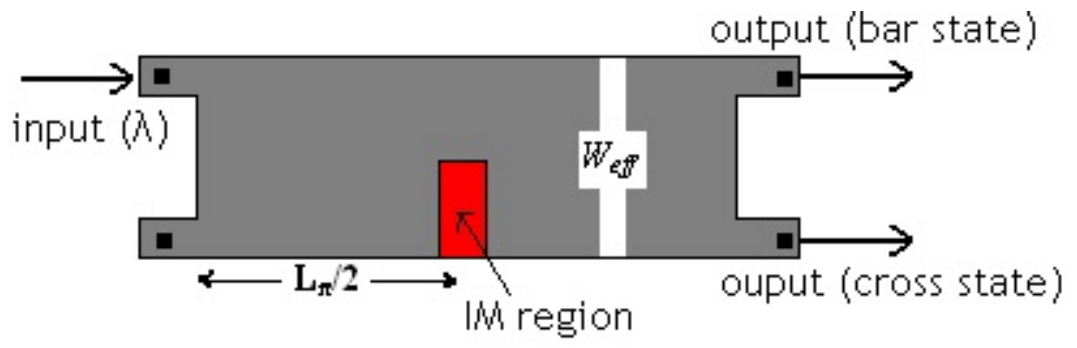

Figure 1: Layout of $2 \times 2$ MMI-switch with IM region, ref. [8]

light to pass through the region. Distribution of the optical intensities in the MMI waveguides occurs in accordance to the beat length, defined as follows. [12]

$$
L_{\pi}=\frac{\pi}{\beta_{0}-\beta_{1}} \approx \frac{4 n_{e f f} W_{e f f}^{2}}{3 \lambda_{0}}
$$

Where $\beta_{0}$ and $\beta_{1}$ are propagation constants of the fundamental and the first-order lateral modes, respectively, $\lambda_{0}$ is a free-space wavelength, $n_{\text {eff }}$ is an effective index, and $W_{\text {eff }}$ is an effective width of the MMI waveguide. The use of different interference phenomena in a device allows achieving different switching states, namely cross and bar. If the width of the MMI regions is reduced by depressing the refractive index by means of the electro-optic (EO) or thermo-optic (TO) effects, then the imaging locations will be changed. Though, EO-effects are highly effective to use in this type of MMI-switch in comparison with TO-effects., as the power consumption is bit higher which further depends upon the length of IM regions.

\section{Modeling of $2 \times 2$ MMI-switch with reconfigurable IM regions}

This section elaborates the design procedure for $2 \times 2$ MMI-switch, which has been tuned by reconfigurable IM regions, while geometries of its various parts are kept very small as compared to. [12-15] Index profile of the channel waveguides is chosen in the range of 2.10-2.20 in agreement with available data and the literature survey to investigate the switching characteristics. Various EO/TO crystals such as Ti-indiffused or proton exchanged lithium niobate $\left(\alpha: \mathrm{LiNbO}_{3}\right)$, lithium tantalate $\left(\mathrm{LiTaO}_{3}\right)$, potassium niobate $\left(\mathrm{KNbO}_{3}\right)$, barium-sodium niobate $(\mathrm{BNN})$, strontium-barium niobate $(\mathrm{SBN})$, bismuth germinates crystals of evlitine structure $\left(\mathrm{Bi}_{4} \mathrm{Ge}_{3} \mathrm{O}_{12}\right)$, lithium iodate $\left(\alpha-\mathrm{LiIO}_{3}\right)$ etc. can be used to implement such channel waveguides. Perfect dimensions for coupling and IM regions have been obtained using the well-known relationship for general interference in MMI waveguides. [6] Combined effect of geometry and refractive index contrast has been applied for modeling of symmetric waveguides to ensure polarization independent propagation.

The switch characteristics are investigated with a tunable laser to generate an input light beam (output level was fixed at $1 \mathrm{~mW}$ and wavelengths were tuned in between $1.3 \mu \mathrm{m}$ and 1.55 
Table 1: Tuning strategies (Structure-1)

\begin{tabular}{|c|c|c|c|c|}
\hline \multirow{2}{*}{ Region } & \multicolumn{3}{|c|}{ Refractive index $\left(n_{1}>n_{2}\right)$} \\
\cline { 2 - 5 } & \multicolumn{2}{|c|}{$1.55 \mu \mathrm{m}$} & \multicolumn{2}{|c|}{$1.3 \mu \mathrm{m}$} \\
\cline { 2 - 5 } & cross & bar & cross & bar \\
\hline Reg.1 & $n_{1}$ & $n_{2}$ & $n_{1}$ & $n_{1}$ \\
\hline Reg.2 & $n_{1}$ & $n_{1}$ & $n_{1}$ & $n_{2}$ \\
\hline Reg.3 & $n_{2}$ & $n_{2}$ & $n_{1}$ & $n_{1}$ \\
\hline
\end{tabular}

$\mu \mathrm{m})$. The various states of the switch are simulated and analyzed using the FD-BPM. [4] Initially we have evaluated the structure by considering less number of IM regions for its satisfactory operation over a bandwidth of $50 \mathrm{~nm}$ for either of centre wavelength $(1.3 \mu \mathrm{m}$ and $1.55 \mu \mathrm{m})$. Fig.2 illustrates the layout of the proposed $2 \times 2 \mathrm{MMI}$-switch labeled as structure- 1 , which represents its $\mathrm{x}-\mathrm{Z}$ slice cut on the wafer of the waveguide with sectional dimensions. The whole device is $500 \mu \mathrm{m}$ long and $8 \mu \mathrm{m}$ wide including IM regions, including $2 \mu \mathrm{m}$ width for upper and lower straight waveguides. The wafer index assumed to be of same value as that of cladding i.e. 1.49 and the thickness of all the regions has been kept same, which is $15 \mu \mathrm{m}$. In this structure, the

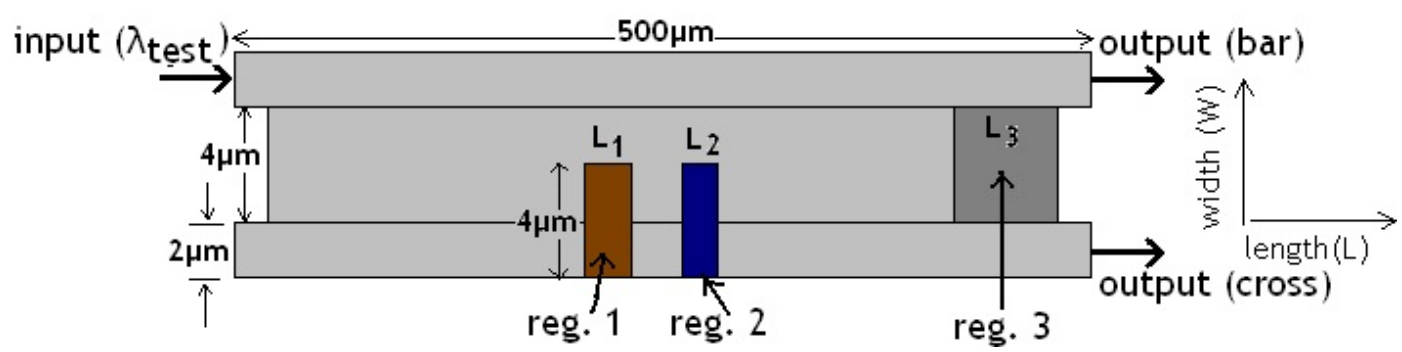

Figure 2: Layout (top view) of the proposed $2 \times 2$ MMI-switch with IM regions (structure-1)

index is modulated for three regions (1 to 3 ) in order to achieve it various switching states in accordance to applied test wavelength. The IM regions represent symmetrical planner multimode waveguides of different area coverage and refractive indices. The size of the index modulated regions are same in terms of their width (4 $\mu \mathrm{m}$ each) but differ in lengths, which are $\mathrm{L}_{1}=12 \mu \mathrm{m}$, $\mathrm{L}_{2}=10 \mu \mathrm{m}$ and $\mathrm{L}_{3}=71 \mu \mathrm{m}$ respectively. Switching in both structures has been achieved by varying the number and locations of reconfigurable IM regions. The refractive index, $\mathrm{n}_{1}$ is kept fixed at 2.22 , while $\mathrm{n}_{2}$ represent reduced index values $\left(\mathrm{n}_{2}=\mathrm{n}_{1}-\delta \mathrm{n}\right)$, centered at 2.136 . The switch performance parameters (propagation losses, CT levels and PDLs) are calculated by altering the value of $\delta \mathrm{n}$, which has been varied in the range of 0.05-0.07.

Table 1 depicts the respective index profile for various IM regions, which are used to tune the switch into its various states. By evaluating this structure it has been noticed that the PDLs observed are at high level for most inputs. In particular, the switch response becomes more sensitive to polarization state of the input for high order wavelengths, which causes imageshifting in the specified IM regions. Therefore few more IM regions are introduced, which can tune to get distinct but clear images for higher order wavelength inputs. Figure 3 illustrate the modified version of previous structure (1), which consists of two additional IM regions termed (4 and 5), each of $4 \hat{\mathrm{Al}} \mathrm{lm}$ wide with a little difference in their length, which are $16.36 \mu \mathrm{m}\left(\mathrm{L}_{4}\right)$ and $16.48 \mu \mathrm{m}\left(\mathrm{L}_{5}\right)$ respectively. Due to this modification, the length of the region 3 is also required 
Table 2: Tuning strategies for modified MMI-switch (structure 2)

\begin{tabular}{|c|c|c|c|c|c|c|c|c|}
\hline Switch State & \multicolumn{4}{|c|}{ Bar state } & \multicolumn{4}{c|}{ Cross state } \\
\hline Mode & \multicolumn{2}{|c|}{ TE } & \multicolumn{2}{c|}{ TM } & \multicolumn{2}{c|}{ TE } & \multicolumn{2}{c|}{ TM } \\
\hline$\lambda(\mu \mathrm{m})$ & 1.55 & 1.3 & 1.55 & 1.3 & 1.55 & 1.3 & 1.55 & 1.3 \\
\hline Reg.1 & $n_{2}$ & $n_{1}$ & $n_{2}$ & $n_{1}$ & $n_{1}$ & $n_{1}$ & $n_{1}$ & $n_{1}$ \\
\hline Reg.2 & $n_{1}$ & $n_{2}$ & $n_{1}$ & $n_{2}$ & $n_{1}$ & $n_{1}$ & $n_{1}$ & $n_{1}$ \\
\hline Reg.3 & $n_{2}$ & $n_{1}$ & $n_{2}$ & $n_{1}$ & $n_{2}$ & $n_{1}$ & $n_{2}$ & $n_{1}$ \\
\hline Reg.4 & $n_{1}$ & $n_{1}$ & $n_{2}$ & $n_{1}$ & $n_{1}$ & $n_{1}$ & $n_{2}$ & $n_{1}$ \\
\hline Reg.5 & $n_{2}$ & $n_{1}$ & $n_{2}$ & $n_{2}$ & $n_{2}$ & $n_{1}$ & $n_{2}$ & $n_{2}$ \\
\hline
\end{tabular}

to reduce by $16.8 \mu \mathrm{m}\left(\mathrm{L}_{3}=54.2 \mu \mathrm{m}\right)$.

However both regions can be made of same length, but in that case, observed PDLs are at higher levels $(\geq 1 \mathrm{~dB})$. Theses extra IM Regions (4 and 5$)$ are defined in order to accommodate TM polarized light images perfectly for either of input wavelength and to suppress the PDLs particularly. The tuning strategies for structure 2 are mentioned in table 2 in details. However

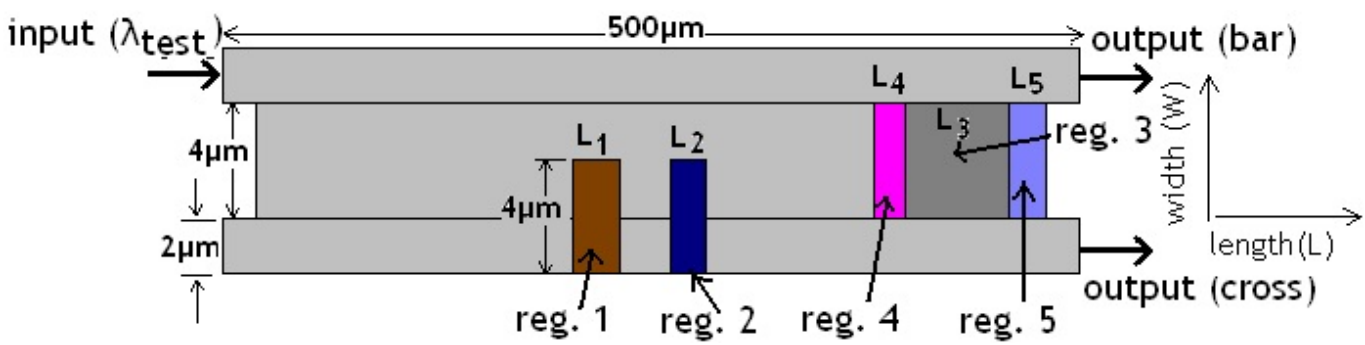

Figure 3: Layout (top view) of the modified $2 \times 2$ MMI-switch for wider operating wavelengths (structure 2)

both regions can be made of same length, but in that case, observed PDLs are at higher levels $(\geq 1 \mathrm{~dB})$. Theses extra IM Regions (4 and 5$)$ are defined in order to accommodate TM polarized light images perfectly for either of input wavelength and to suppress the PDLs particularly. The tuning strategies for structure 2 are mentioned in table 2 in details.

Figures $4(\mathrm{a}-\mathrm{c})$ and 5 (a-c) shows a comparison of calculated E.L., I.L. and corresponding CT levels for both structures. While these are evaluated for TM polarized input of a test wavelengths of $1.55 \mu \mathrm{m}$ and $1.3 \mu \mathrm{m}$. From these figures, it is clear that by introducing an extra IM region (Region 5) within the coupling section, as in case of structure 2, switch can be made to operate with reduced losses and better CT levels even for TM polarized optical inputs. Plots of figure 4 clearly indicate that with modified structuring, reduction in the switch losses i.e. E.L. and I.L. of the order of $2.25 \mathrm{~dB}$ and $2.50 \mathrm{~dB}$ with a reduction in the CT levels up to $5.8 \mathrm{~dB}$ can be achieved. Similarly trends in plots of figure 5, gives an indication of suppression in the switch losses (of the order of $1 \mathrm{~dB}$ ) at a cost of increased CT levels by $1 \mathrm{~dB}$ for modified structure case.

Similarly plots in figure 6 (a-c) shows a comparison of calculated E.L., I.L. and corresponding $\mathrm{CT}$ levels for TE/TM polarization case for modified structure 2, while the optical test wavelength is $1.55 \mathrm{~A} l \mathrm{l} m$. From these plots, it is very clear that within modified structure, PDL of less than 0.15 in any case can be maintained, while the switch losses in worst case remains below $0.8 \mathrm{~dB}$ and the best $\mathrm{CT}$ level of $-23.5 \mathrm{~dB}$ can be achieved. Again with a slight variation in $\delta \mathrm{n}$, the switch losses unaffected at large. However CT levels are more sensitive and tends to increase to 


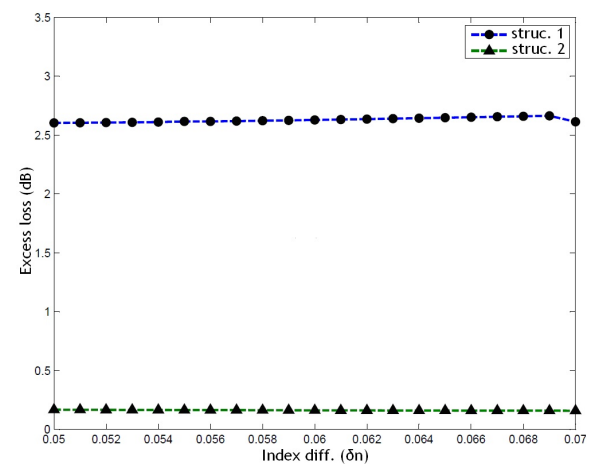

(a)

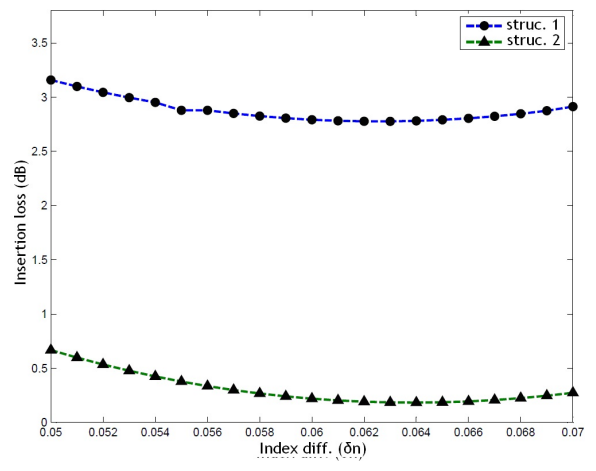

(b)

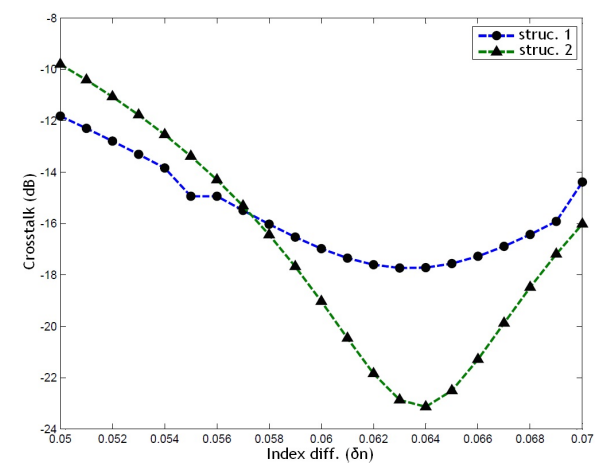

(c)

Figure 4: Calculated variation in the (a) E.L. (b) I.L. and (c) CT levels with respect to index variations $(\delta \mathrm{n})$, while the switches (Struct. 1 and 2) are in bar state, while subjected to a TM polarized optical input of the test wavelength $(\lambda)$ of $1.55 \mu \mathrm{m}$

higher levels in case of TM polarized inputs, thereby affecting the switching performance badly. Similar trends for test input wavelength of $1.3 \mu \mathrm{m}$ have been noticed depicted by plots of figure 7 (a-c), which are showing a comparison of calculated E.L., I.L. and corresponding CT levels for TE/TM polarization case for modified structure. From these plots, it is clear that with modified structuring, PDLs of less than 0.07 in either case of test wavelength can be maintained, while the structure possess switching losses $\leq 1.1 \mathrm{~dB}$ with a best value for $\mathrm{CT}$ of $-23.8 \mathrm{~dB}$.

By evaluating both structures, it has been noticed that by employing extra IM regions, the MMI switch can be made to operate with reduced losses and better CT levels irrespective of polarization state of the inputs. It is also noticed later that for modified structure (2), PDL $\leq 0.15 \mathrm{~dB}$ in any case can be maintained, while in worst case, the switch possess propagation losses $\leq 0.8 \mathrm{~dB}$ with a best possible $\mathrm{CT}$ level of $-23.5 \mathrm{~dB}$. With modified structure, suppression in the switch losses (E.L., I.L.) of $1 \mathrm{~dB}$ approximately can be achieved. Table 3 summarizes the performance parameters for both considered structures. With this table, one can predict that performance of such switching structure can be optimize further by introducing more IM regions, to generate clear, concentrated and lossless images within the coupling region. However insertion of more IM regions will affect the design complexity, tolerance factors and cost of the operation.

\section{Conclusion}

MMI waveguides based small size $2 \times 2$ photonic switches are realized. Concept of self-imaging in MMI waveguides has been used to introduce reconfigurable IM regions in a high refractive index 


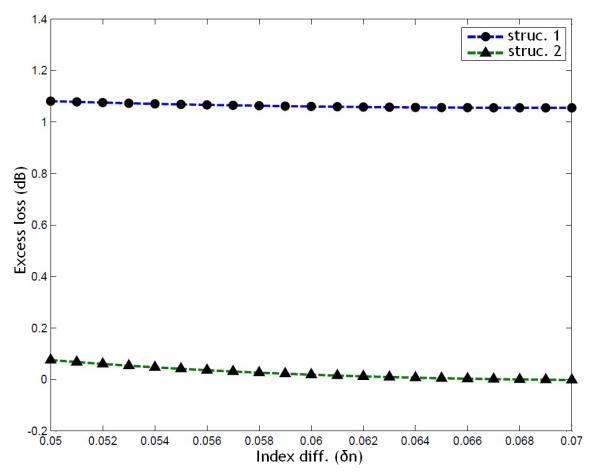

(a)

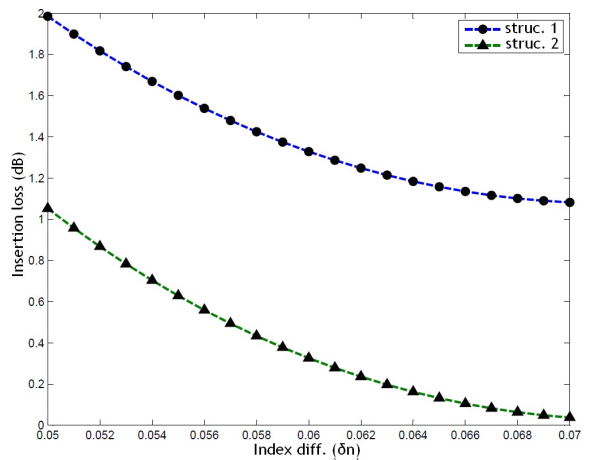

(b)

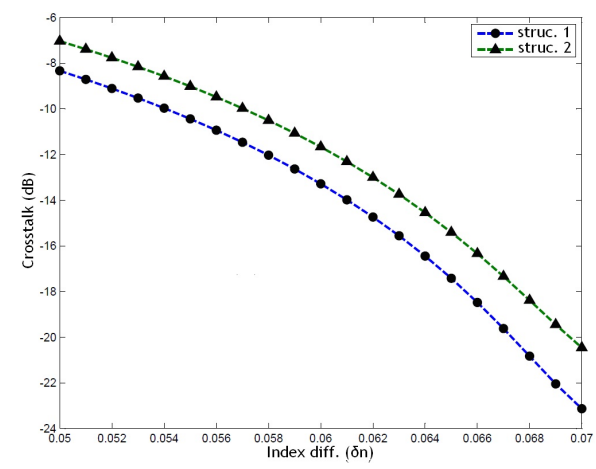

(c)

Figure 5: Calculated variation in the (a) E.L. (b) I.L. and (c) CT levels with respect to index variations $(\delta \mathrm{n})$, while the switches (Struct. 1 and 2) are in bar state, while subjected to a TM polarized optical input of the test wavelength $(\lambda)$ of $1.3 \mu \mathrm{m}$

contrast to tune the switch for different wavelengths. It is observed that with introduction of more IM regions, switch losses, corresponding CT and PDL levels can be suppressed significantly. The smaller dimensions of proposed structures shall provide an opportunity to reducing fabrication costs and increasing the density in optical networks. Therefore such structures can be proven as suitable to realize higher order switches due to their advantages of minimum propagation losses, better CT levels and ease of extendibility into a multi-port configuration. Also their compact nature with higher tolerance to avoid fabrication errors shall makes them promising elements for photonic integrated circuits (PIC) and monolithic functional switches. 


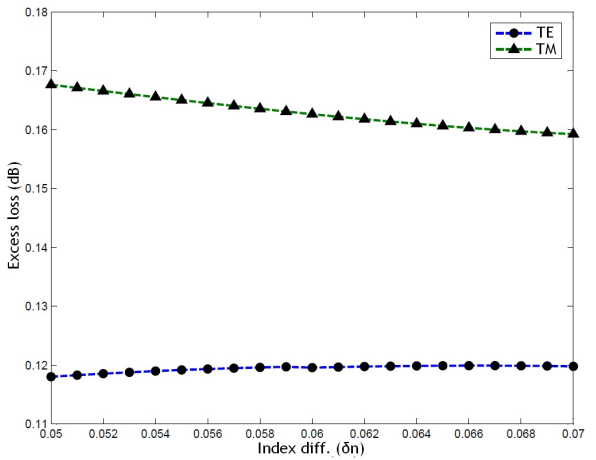

(a)

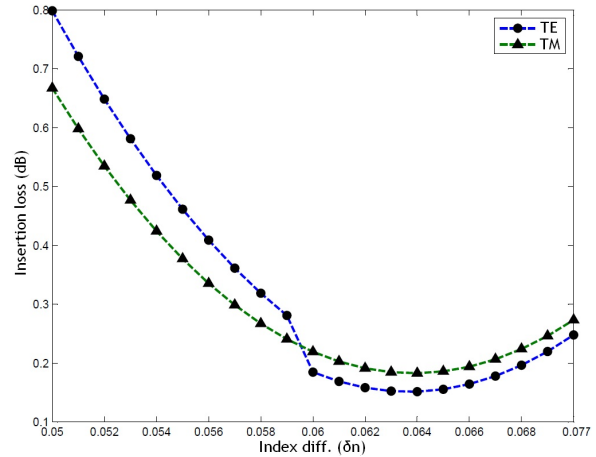

(b)

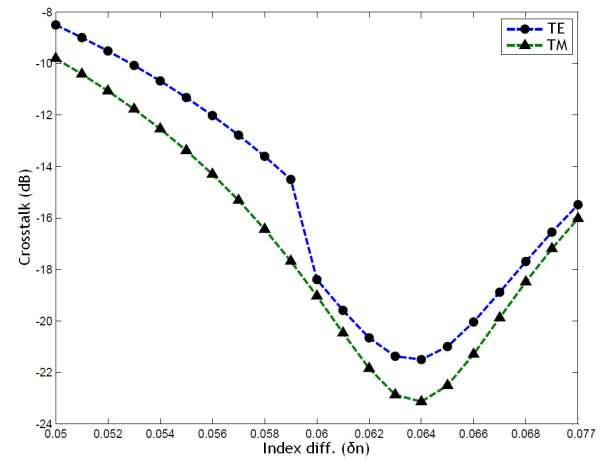

(c)

Figure 6: Calculated variation in the (a) E.L. (b) I.L. and (c) CT levels with respect to index variations $(\delta \mathrm{n})$, while the switch (Structure-2) is in bar state and subjected to a TE/TM polarized optical inputs at test wavelength $(\lambda)$ of $1.55 \mu \mathrm{m}$

Table 3: Comparative summary of performance parameters

\begin{tabular}{|c|c|}
\hline \multicolumn{2}{|r|}{ Performance parameters (structure 1) } \\
\hline$\lambda_{\text {test }}: 1.3 \mu m$ & $\begin{array}{c}\text { E.L. } \leq 1.1 \mathrm{~dB}(\mathrm{TM}) \text { and } \leq 0.07 \mathrm{~dB}(\mathrm{TE}) \\
\text { I.L. } \leq 2 \mathrm{~dB}(\mathrm{TM}) \text { and } \leq 0.8 \mathrm{~dB}(\mathrm{TE}) \\
\mathrm{CT}(\text { best value }):-23.4 \mathrm{~dB}(\mathrm{TE}),-23.2 \mathrm{~dB}(\mathrm{TM}) \\
\text { PDL }_{\text {max. }}: \leq 0.1 \mathrm{~dB}\end{array}$ \\
\hline$\lambda_{\text {test }}: 1.55 \mu \mathrm{m}$ & $\begin{array}{c}\text { E.L. } \leq 2.7 \mathrm{~dB}(\mathrm{TM}) \text { and } \leq 0.12 \mathrm{~dB}(\mathrm{TE}) \\
\text { I.L. } \leq 3.3 \mathrm{~dB}(\mathrm{TM}) \text { and } \leq 0.8 \mathrm{~dB}(\mathrm{TE}) \\
\text { CT (best value): }-21.8 \mathrm{~dB}(\mathrm{TE}) \text { and }-17.4 \mathrm{~dB}(\mathrm{TM}) \\
\text { PDL }_{\text {max. }}: \leq 2.6 \mathrm{~dB}\end{array}$ \\
\hline & Derformance parameters (structure 2) \\
\hline$\lambda_{\text {test }}: 1.3 \mu m$ & $\begin{array}{c}\text { E.L. } \leq 0.08 \mathrm{~dB}(\mathrm{TM} / \mathrm{TE}) \\
\text { I.L. } \leq 1.2 \mathrm{~dB}(\mathrm{TM} / \mathrm{TE}) \\
\text { CT (best value) }:-23.8 \mathrm{~dB}(\mathrm{TE}) \text { and }-20.2 \mathrm{~dB}(\mathrm{TM}) \\
\text { PDL }_{\text {max. }} \leq \leq 0.07 \mathrm{~dB}\end{array}$ \\
\hline$\lambda_{\text {test }}: 1.55 \mu \mathrm{m}$ & $\begin{array}{c}\text { E.L. } \leq 0.17 \mathrm{~dB}(\mathrm{TM} / \mathrm{TE}) \\
\text { I.L. } \leq 0.8 \mathrm{~dB}(\mathrm{TM} / \mathrm{TE}) \\
\text { CT (best value) }:-21.8 \mathrm{~dB}(\mathrm{TE}) \text { and }-23.3 \mathrm{~dB}(\mathrm{TM}) \\
\text { PDL }_{\max .}: \leq 0.15 \mathrm{~dB}\end{array}$ \\
\hline
\end{tabular}




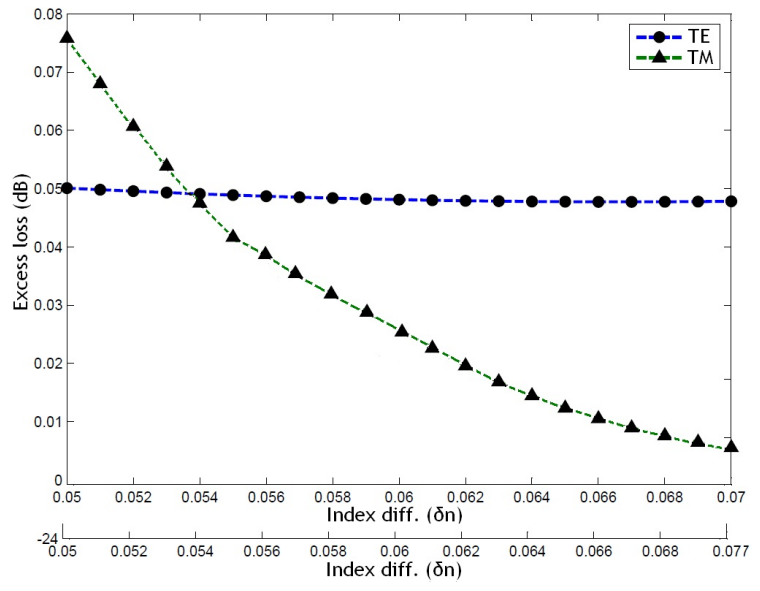

(a)

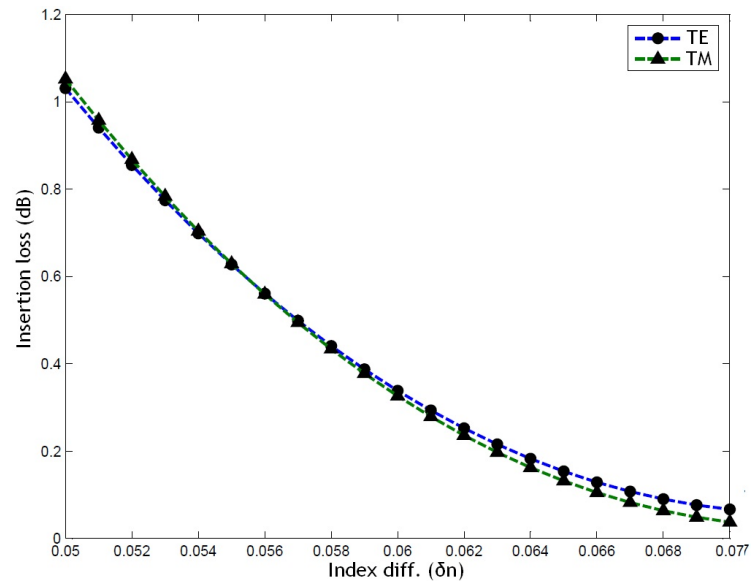

(b)

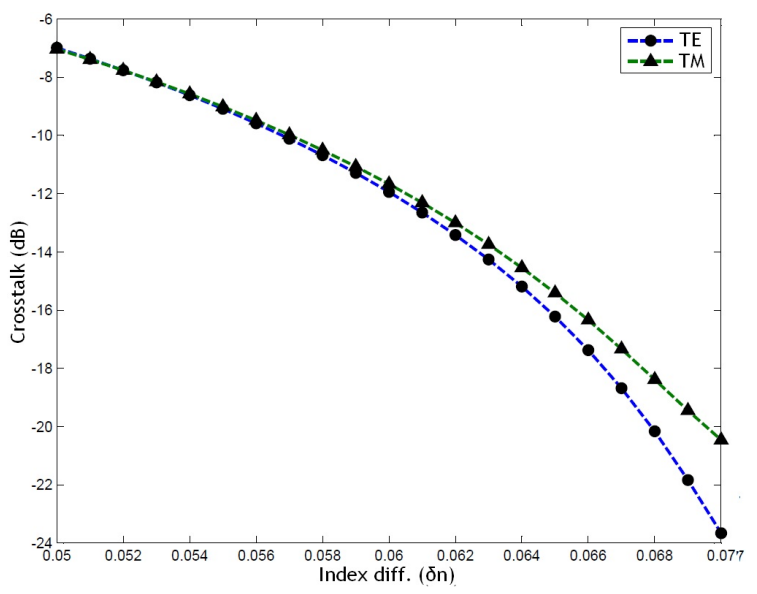

(c)

Figure 7: Calculated variation in the (a) E.L. (b) I.L. and (c) CT levels with respect to index variations $(\delta \mathrm{n})$, while the switch (Structure-2) is in bar state and subjected to a TE/TM polarized optical inputs at test wavelength $(\lambda)$ of $1.3 \mu \mathrm{m}$ 


\section{Bibliography}

[1] S. Kumai, T. Ishikawa, A. Okazaki, K. Utaka, et al., High-speed optical switching of InAlGaAs/InAlAs multimode interference photonic switch with partial index-modulation region (MIPS-P), IEICE Electron. Express, 2(23):578-582, 2005.

[2] Z. Jin, G. Peng, Designing optical switches based on silica MMI devices, Progress in Electromagnetic Research Symposium, Hangzhou, China, pp. 58-61, August 22-26, 2005.

[3] X. Wu, L. Liu, Y. Zhang, et al., Low electric power drived thermo-optic MMI-switches with tapered heating electrodes, Optics Communications, 258: 135-143, 2006.

[4] X.Q. Sun, C.M. Chen, et al., A MMI polymer-silica hybrid waveguide $2 \times 2$ thermo-optic switch, Optica Applicata, vol. xl, no. 3, 2010.

[5] L. Cahill, The modeling of MMI devices, Proc. ICTON, ieeexplorer, vol. 2, pp. 138-141, Nottingham, U.K., June, 2006.

[6] P. P. Sahu, A tapered structure for compact MMI-coupler, IEEE Photon. Technol. Lett., 20(8):638-640, April 15th, 2008.

[7] K. Solehmainen, M. Kapulainen, M. Harjanne, T. Aalto, Adiabatic and MMI-couplers on SOI, IEEE Photon. Technol. Lett., 18(21), Nov. 1st, 2006.

[8] A. M. Al hetar, A. S. M. Supaat, A. B. Mohammad, I. Yulianti, MMI photonic switches, Optical Engineering, 47(11), 112001, Nov., 2008.

[9] A. M. Al-Hetar, A. B. Mohammad et al., MI-MZI polymer thermo-optic switch with a high refractive index contrast, Journal of Lightwave Technology, 29(2):171-178, January 15th, 2011.

[10] L. B. Soldano, E. C. M. Pennings, Optical multimode interference devices based on selfimaging: Principles and applications, Journal of Lightwave Tech., 13(4):615-627, 1995.

[11] R. Ulrich, Image formation by phase coincidences in optical waveguides, Opt. Comm., 13:259-264, 1975.

[12] S. Nagai, G. Morishima, H. Inayoshi, K. Utaka, Multimode Interference Photonic Switches (MIPS), Journal of Lightwave Technology, 20(4):675-681, April, 2002.

[13] Z. Jin, G. Peng, Designing optical switches based on silica MMI devices, Proc. of progress in Electromagnetic Research Symposium, Hangzhou, China, pp. 58-61, August 22-26, 2005.

[14] F. Wang, J. Yang, L. Chen, X. Jiang, M. Wang, Optical switch based on MMI-coupler, IEEE Photon. Technol. Lett., 18 (2):421-423, 2006.

[15] D. A. M. Arrioja, N. Bickel, P. Likamwa, Robust $2 \times 2$ MMI optical switch, Optical and Quantum Electronics, 38:557-566, 2006. 